that individuals and institutions should persistently and incrementally strive to do the best they can do to reduce HAIs and improve patient safety are more likely to achieve acceptance by staff who have varying roles and priorities and numerous other jobrelated concerns.

We also need to recognize that our efforts to reduce antimicrobial resistance should be tempered with the reality that many of its causes are not amenable to direct intervention by individual doctors, healthcare systems, or government agencies. Burdening clinicians with this responsibility or blaming them when resistance gets worse makes our job harder when we recommend partially effective but still useful changes in policies, protocols, and care practices. Instead, we may have more credibility and impact by focusing on explaining and attempting to address basic principles, the relationships between colonization and subsequent infection, the role of local antibiotic pressure and local emergence of resistance, and the adverse risks of devices.

We should endorse Easterby's recommendation that activists need to listen to their doubters and resist, as best they can, temptations to lambaste them when their opinions clash with ours. Hospital epidemiologists who occasionally disparage colleagues who doubt, ignore, or are indifferent to our efforts are more likely to be frustrated than successful.

Also, we need to fix "computer models" overly reliant on flawed surveillance definitions. It is often counterproductive to rely on metrics and outcomes such as C. difficile "lab ID events" that currently cannot reliably distinguish between true infection and colonization or endorsing and using flawed definitions of a catheter-associated bloodstream or urinary tract infection. Many of our colleagues are skeptical because they correctly realize that use of these metrics to assess and monitor the impact and efficacy of specific prevention protocols and policies is often misleading. We need to develop surveillance definitions that are clinically accurate; comprehensible to clinicians; and have clear impact on clinical practice, quality, and safety. Otherwise, we will continue to experience the same skepticism and indifference that climate activists encounter when then rely on unintelligible and sometimes inaccurate National Oceanic and Atmospheric Administration climate data.

Finally and most importantly: how can hospital epidemiologists become better salesmen? For starters, we can collectively and individually alter prior behaviors and approaches that have led to failure. We should stop assuming that apathy or ignorance of our physician and nursing colleagues are behind the frequent failure of our protocols, policies, and recommendations. All of us need to become better versed and trained in the arts of salesmanship, negotiations, active listening, communication and even marketing. Multiple on-line and on-site training programs are available for developing these and standard business and sales skills. Some of us could benefit from hiring a personal coach to provide individual help and feedback. We can also learn by observing and mimicking effective people who understand and are skilled in sales and marketing. Our society should be urged to collectively and publicly petition The Centers for Disease Control to revamp or even abandon flawed surveillance definitions. Our society should stop overt or tacit support of the use of inaccurate and flawed metrics to punish hospitals. We need to realize that many of our prior efforts have failed because we, too, lack "clarity, credibility, and empathy" in dealing with our fellow healthcare brethren.

Although effective hospital epidemiologists utilize numerous other "tools and assets," such as negotiation and complex strategies requiring flexibility, compromises, relationship building, and priority setting, salesmanship is too often underutilized. But salesmanship alone will never be a panacea. Even if we achieve reasonable competency in the preceding skills and techniques, we will still periodically encounter failure, frustration and disappointment. And when these failures and frustrations occur, I advise making our best effort to sustain our focus and retain our optimism and goals while pondering Shakespeare's famous line: "The fault, dear Brutus, is not in our stars but in ourselves, that we are underlings.”

\section{Acknowledgments.}

Financial support. No financial support was provided relevant to this article.

Conflicts of interest. The authors reports no conflicts of interest relevant to this article.

\section{References}

1. Easterby S. Climate activists are lousy salesman. The Wall Street Journal, April 25, 2016.

2. Price JR, Golubchik T, Coke $\mathrm{K}$, et al. Whole genomic sequencing shows that patient-to-patient transmission rarely accounts for acquisition of Staphylococcus aureus in an intensive care unit. Clin Infect Dis 2014;58:609-618.

3. Eyre DW, Cule M, Wilson DJ, et al. Diverse sources of $C$. difficile infection on whole-genomic sequencing. N Engl J Med 2013;369:1195-1205.

4. Palmore TN, Henderson DK. Managing transmission of carbapenemresistant Enterobacteriaceae in healthcare settings: a view from the trenches. Clin Infect Dis 2013;57:1593-1599.

\title{
Strict sequestration versus lenient isolation precautions during hematopoietic stem cell transplant: results of a quality initiative
}

\author{
Joseph K. Franz $M D^{1}$, Alexander R. Coltoff $M D^{1}$ and Amir S. Steinberg $M D^{2}$ \\ ${ }^{1}$ Department of Internal Medicine, Icahn School of Medicine at Mount Sinai, New York, New York and ${ }^{2}$ Division of Hematology and Oncology, Mount Sinai \\ Hospital, New York, New York
}

Author for correspondence: Joseph Franz MD, One Gustave L. Levy Place, Bod 1118, New York, NY 10029. E-mail: Joseph.franz@mountsinai.org

Cite this article: Franz Jk. et al. (2018). Strict sequestration versus lenient isolation precautions during hematopoietic stem cell transplant: results of a quality initiative. Infection Control \& Hospital Epidemiology 2018, 39, 1270-1272. doi: 10.1017/ice.2018.203
To the Editor-Inpatient bone marrow transplant (BMT) requires long hospitalization on the order of weeks to months. During this time, hematopoietic stem cell transplant (HSCT) recipients are at 
risk for disease- and treatment-related complications, and neutropenic infections are a significant cause of morbidity and mortality. Long hospitalizations are burdensome to patients, both physically and mentally, and remaining sedentary leads to physical deconditioning that often necessitates physical rehabilitation either during hospitalization or after discharge. ${ }^{1}$ Additionally, the decreased quality of life and stress during the transplant hospitalization has been associated with the development of depression and posttraumatic stress disorder (PTSD). ${ }^{2}$

Practice guidelines for antibiotic prophylaxis and treatment of neutropenic infections are well-established. ${ }^{3}$ Although there are guidelines for nonpharmacologic infection prevention measures, there is little evidence to support a standard of care, and practices vary by institution. Commonly accepted practices include singleoccupant rooms with HEPA filtration systems, strict hand hygiene, and the avoidance of fresh fruits, vegetables and plants etc. ${ }^{4}$ Recent literature has also suggested that surgical masks for all persons in contact with neutropenic patients can reduce the incidence of viral respiratory infections (VRIs). ${ }^{5}$ Although it is a common policy for patients to be under strict isolation precautions during periods of prolonged neutropenia, there remains insufficient evidence to support this practice. ${ }^{6}$

Prior to October 2016, neutropenic patients were sequestered in private rooms on the BMT unit at our institution. During routine review, physical therapists noted that sequestered patients were overly sedentary and susceptible to physical deconditioning during this time. In response to these concerns, the Bone Marrow Transplant Quality Assurance Committee lifted the sequestration order, allowing neutropenic patients on the BMT unit to leave their rooms if they maintained strict handwashing and surgical mask precautions. We hypothesized that this change in policy would reduce both the need for physical therapy (PT) and hospital length of stay (LOS), as well as improve overall patient satisfaction. These benefits were believed to outweigh the theoretical increased risk of infection associated with leaving an isolated environment.

We reviewed 143 records of sequentially admitted patients before the neutropenic policy before the neutropenic policy change from February 2016 through September 2016 and 188 records after the policy was changed from October 2016 through June 2017. The inclusion criterion was admission to the BMT unit for autologous or allogeneic stem cell transplant, regardless of indication.

Hospital LOS and overall PT requirements were recorded. PT requirements were determined based upon a physical therapist's recommendation and included acute rehabilitation, subacute rehabilitation, or home PT. The frequency of PT consultation was also recorded. The total number of documented hospital-acquired VRIs, as diagnosed by a comprehensive viral panel polymerase chain reaction (PCR), was recorded in each study group. Viral respiratory infections were diagnosed as hospital-acquired infections retrospectively if patients were asymptomatic on admission, and PCR was confirmed after the estimated incubation period of the virus. ${ }^{7-10}$

Baseline characteristics are summarized in Table 1. The average hospital LOS was 19.00 days in the sequestered group and was 19.31 days in the nonsequestered group (2-proportion $\mathrm{z}$ test, $\mathrm{z}=.74 ; P=.46)$. There were 50 PT consults in the sequestered group and 53 consults in the nonsequestered group (2-proportion $\mathrm{z}$ test, $\mathrm{z}=1.32 ; P=.19)$. Physical therapy was recommended for 19 patients in the sequestered group and 34 patients in the nonsequestered group (2-proportion $\mathrm{z}$ test, $\mathrm{z}=.99 ; P=.32$ ). During the study period, 1 VRI occurred in the sequestered group
Table 1. Baseline Characteristics

\begin{tabular}{lcc}
\hline Baseline Characteristics & $\begin{array}{c}\text { Sequestered } \\
\text { Group } \\
(\mathrm{N}=143)\end{array}$ & $\begin{array}{c}\text { Nonsequestered } \\
\text { Group } \\
(\mathrm{N}=188)\end{array}$ \\
\hline Age median (range), y & $59(23-83)$ & $58(19-74)$ \\
\hline Male, no. (\%) & $76(53.1)$ & $98(52.1)$ \\
\hline Type of transplant, no. (\%) & $124(86.7)$ & $151(80.3)$ \\
\hline Autologous & $19(13.3)$ & $37(19.7)$ \\
\hline Allogeneic & $108(75.5)$ & $130(69.1)$ \\
\hline Indication for Transplant, no. (\%) & $13(9.1)$ & $12(6.4)$ \\
\hline Multiple myeloma & $8(5.6)$ & $6(3.2)$ \\
\hline Non-Hodgkin Lymphoma & $6(4.2)$ & $30(16.0)$ \\
\hline Acute myeloid leukemia & $8(5.6)$ & \\
\hline Acute lymphoblastic leukemia & & \\
\hline Other & & $10.3)$ \\
\hline
\end{tabular}

and 3 occurred in the nonsequestered group (2-proportion $\mathrm{z}$ test, $\mathrm{z}=.74 ; P=.46)$. We recorded 1 case of each of the following VRIs: influenza A, respiratory syncytial virus (RSV), metapneumovirus, and parainfluenza 3.

There is insufficient evidence to support the common practice of patient sequestration during post-HSCT neutropenia. While sequestration, in theory, reduces infection risk, it is potentially harmful because it may exacerbate physical deconditioning and cause psychological distress. Our findings do not support our original hypothesis that lifting the strict neutropenic isolation policy would decrease PT requirements and hospital LOS. A possible explanation for this finding is the inability to verify that the nonsequestered patients did, in fact, ambulate outside of their rooms. Given the well-known risk of infection associated with HSCT, many patients may have elected to stay in their rooms out of fear of acquiring an infection. Additionally, the degree of physical deconditioning associated with BMT patients and the benefit of ambulating around the unit versus ambulating in the hospital room may have been overestimated.

Although we observed was no difference in PT requirements and hospital LOS, we also observed no significant difference in incidence of VRIs between the 2 groups. Notably, the sequestration policy changed in October, so the nonsequestered group was studied over more winter months, when the overall incidence of VRIs is higher. Although the study population and incidence of VRI was small, these data suggest that the risk of acquiring a VRI during neutropenia by leaving an isolated room may be small and may potentially be outweighed by the benefits, namely, the psychological benefits of leaving confinement. A future study with a large sample size could better assess this risk and could focus on the psychological implications of sequestration and the benefits of ambulation outside of the hospital room.

In conclusion, more lenient neutropenic isolation precautions were not associated with decreased PT requirements or hospital LOS but were also not associated with an increased rate of hospital-acquired VRIs. 


\section{Acknowledgments.}

Financial support. No financial support was provided relevant to this article.

Conflicts of interest. All authors report no conflicts of interest relevant to this article.

\section{References}

1. Hayes SC, Davies PS, Parker TW, Bashford J, Green A. Role of a mixed type, moderate intensity exercise programme after peripheral blood stem cell transplantation. Br J Sports Med 2004;38:304-309.

2. El-Jawahri A. R., Vandusen H. B., Traeger L. N. et al. Quality of life and mood predict posttraumatic stress disorder after hematopoietic stem cell transplantation. Cancer 2016;122:806-812.

3. Freifeld AG, Bow EJ, Sepkowitz KA, et al. Clinical practice guideline for the use of antimicrobial agents in neutropenic patients with cancer: 2010 update by the Infectious Diseases Society of America. Clin Infec Dis 2011;52:e56-e93.

4. Tomblyn M, Chiller T, Einsele H, et al. Guidelines for preventing infectious complications among hematopoietic cell transplantation recipients: a global perspective. Biol Blood Marrow Transpl 2009;15:1143-1238.
5. Sokol KA, De La Vega-Diaz I, Edmondson-Martin K, et al. Masks for prevention of respiratory viruses on the BMT unit: results of a quality initiative. Transpl Infect Dis 2016;18:965-967.

6. Hayes-Lattin B, Leis JF, Maziarz RT. Isolation in the allogeneic transplant environment: How protective is it? Bone Marrow Transpl 2005;36: 373-381.

7. Interim guidance on the use of influenza antiviral agents during the 20102011 influenza season. US Centers for Disease Control and Prevention website. http://www.cdc.gov/flu/professionals/antivirals/guidance/summary.htm. Published 2012. Accessed on March 13, 2018.

8. American Academy of Pediatrics. Respiratory syncytial virus. In: Red Book: 2015 Report of the Committee on Infectious Diseases, $30^{\text {th }}$ Edition, Kimberlin DW, Brady MT, Jackson MA, Long SS, eds. Elk Grove Village, IL: American Academy of Pediatrics; 2015.

9. Centers for Disease Control and Prevention (CDC). Outbreaks of human metapneumovirus in two skilled nursing facilities-West Virginia and Idaho, 2011-2012, MMWR Morb Mortal Wkly Rep 2013;62:909-913.

10. American Academy of Pediatrics. Parainfluenza viral infections. In: Red Book: 2015 Report of the Committee on Infectious Diseases, $30^{\text {th }}$ Edition, Kimberlin DW, Brady MT, Jackson MA, Long SS, editors. Elk Grove Village, IL: American Academy of Pediatrics; 2015.

\title{
Impact of elimination of contact precautions on noninfectious adverse events among MRSA and VRE patients
}

\author{
Sumanth Gandra MD ${ }^{1,2}$, Constance M. Barysauskas $\mathrm{MS}^{3}$, Deborah A. Mack RN ${ }^{4}$, Bruce Barton PhD ${ }^{5}$, Robert Finberg \\ $M D^{6}$ and Richard T. Ellison $M D^{6}$ \\ ${ }^{1}$ Medical Microbiology Fellow, Department of Pathology, University of Chicago, Chicago, Illinois, ${ }^{2}$ NorthShore University Health System, Evanston Hospital, \\ Evanston, Illinois, ${ }^{3}$ Veristat, Southborough, Massachusetts, ${ }^{4}$ Infection Control Department, University of Massachusetts Medical Center, Worcester, \\ Massachusetts, ${ }^{5}$ Quantitative Health Sciences, University of Massachusetts Medical School, Worcester, Massachusetts and and ${ }^{6}$ Division of Infectious Disease \\ and Immunology, University of Massachusetts Medical School, Worcester, Massachusetts
}

To the Editor-We read with great interest the article by Martin et $\mathrm{al}^{1}$ published online in May 2018 in Infection Control and Hospital Epidemiology. ${ }^{1}$ We previously reported on the impact of elimination of contact precautions (CP) in methicillin-resistant Staphylococcus aureus (MRSA) and vancomycin-resistant enterococci (VRE) patients on noninfectious complications, although our analysis was limited to falls and pressure ulcers. ${ }^{2}$ Our findings differed from those of Martin et al; we observed no statistically significant difference in the rate of falls or pressure ulcers among MRSA/VRE patients in the years before and after eliminating CP. The rate of falls among MRSA/VRE patients in the year before eliminating CP was 4.57 per 1,000 patient days, and it was 4.82 per 1,000 patient days in the year after eliminating contact precautions $(P=074)$. Similarly, the rate of pressure ulcers in the year before eliminating $\mathrm{CP}$ was 4.87 per 1,000 patient days, and it was 4.17 per 1,000 patient days in the year after eliminating contact precautions $(P=.33)$.

Martin et al report a significant drop in the number of noninfectious adverse events among MRSA/VRE patients in the year

Author for correspondence: Sumanth Gandra MD, Room 1917, Evanston Hospital, 2650 Ridge Avenue, Evanston, IL 60201. E-mail: gandrasatyam@gmail.com

Cite this article: Gandra S, et al. (2018). Impact of elimination of contact precautions on noninfectious adverse events among MRSA and VRE patients. Infection Control \& Hospital Epidemiology 2018, 39, 1272-1273. doi: 10.1017/ice.2018.204 after eliminating CP (21.4 per 1000 admissions vs 6.08 per 1000 admissions; $P<.001$ ). In contrast to our study, the study summarized by Martin et al not only included falls and pressure ulcers but also hemorrhage, postoperative respiratory failure, wound dehiscence, and pulmonary embolism or deep vein thrombosis. Although the composite index of all noninfectious adverse events showed a significant drop, the authors did not present a breakdown by individual adverse events in MRSA/VRE and nonMRSA/VRE patients.

We reported 2 additional important findings in our study. First, MRSA/VRE patients had a statistically significant higher Charlson comorbidity index (CCI) compared with non-MRSA/VRE patients (mean CCI, 3.32 vs $2.75 ; P=.002$ ). This was not examined by Martin et al. Second, compared to non-MRSA/VRE patients, we found that MRSA/VRE patients had significantly higher rates of falls (4.57 per 1,000 patient days vs 2.04 per 1,000 patient days) and pressure ulcers (4.87 per 1,000 patient days vs 1.22 per 1,000 patient days), both in the year before and in the year after eliminating CP. Based on figure 2 from Martin EM et al, the rate of noninfectious adverse events were much higher in MRSA/VRE patients than in non-MRSA/VRE patients in the year prior to elimination of CP. However, in the year thereafter, there seems to be no difference.

The reason for the discrepancy in the findings between the 2 studies is unclear. However, we have identified 2 differences between 